\title{
Approche du déterminisme du rythme de croissance endogène des jeunes chênes pédonculés par modulation de l'intensité lumineuse
}

\author{
P Barnola 1, D Alatou 2, C Parmentier 1, C Vallon 1 \\ 1 Laboratoire de Biologie des ligneux, Faculté des Sciences, \\ BP 239, 54506 Vandouvre Cedex, France; \\ 2 Institut des Sciences de la Nature, Université de Constantine, Constantine, Algérie
}

(Reçu le 20 octobre 1992; accepté le 10 février 1993)

\begin{abstract}
Résumé - À l'obscurité, la croissance rythmique est déstabilisée, elle devient continue. Le marquage par la $\left[{ }^{14} \mathrm{C}\right]-D M O$ montre que le bourgeon terminal est le centre utilisateur dominant. L'expression temporelle du rythme, à l'inverse de l'expression spatiale, subit des variations importantes lorsque les plantes sont cultivées avec une alternance lumière/obscurité. De très faibles intensités lumineuses sont capables de restaurer la croissance rythmique de plantes initialement cultivées à l'obscurité. Si les fortes intensités lumineuses ne modifient pas les caractéristiques de la croissance rythmique endogène, elles favorisent l'installation de la ramification. La croissance rythmique endogène est un processus complexe où l'expression temporelle, aisément malléable, est vraisemblablement en grande partie sous l'influence de corrélations à longue distance et l'expression spatiale, très stable, dépendante de corrélations à courte distance extra- et intraapicales. L'hypothèse est faite selon laquelle l'allocation glucidique occuperait une place importante dans les corrélations impliquées dans la croissance rythmique endogène du chêne pédonculé.
\end{abstract}

croissance rythmique / expression spatiale / expression temporelle / chêne pédonculé / obscurité / intensitê lumineuse

Summary - Study of the determinism of endogenous rhythmic growth of young common oak by light modulation. In darkness, rhythmic growth is disturbed and becomes continuous. $\left[{ }^{14} \mathrm{C}\right]-$ DMO labelling shows that the apical bud is the main sink. Unlike the spatial component, the temporal component undergoes strong variations when plants are grown under alternating light and dark conditions.

Very weak light intensities are able to restore rhythmic growth of plants initially grown under dark conditions. Strong light intensities do not change endogenous rhythmic growth characteristics but allow branching.

Endogenous rhythmic growth is an intricate problem where the rather labile temporal component is probably largely under the influence of long-distance correlations and the very stable spatial component is dependent on extra- and intra-apical short-distance correlations. The glucidic allocation appears to be important in the correlative control of endogenous rhythmic growth in Quercus robur.

rhythmic growth / spatial component / temporal component / common oak / darkness / light intensity 


\section{INTRODUCTION}

Le chêne pédonculé, espèce forestière majeure des zones tempérées européennes, est un modèle dont l'intérêt est certain pour l'étude du déterminisme de la croissance rythmique des ligneux (Barnola et al, 1986; Champagnat et al, 1986; Alatou et al, 1989). Le rythme est de nature endogène (Lavarenne-Allary, 1965). Cultivé en jour long ou continu sous une intensité de $81 \mu \mathrm{E} \cdot \mathrm{m}^{-2} \cdot \mathrm{s}^{-1}$, à une température constante de $25^{\circ} \mathrm{C}$, il présente une succession ininterrompue de vagues de croissance ou flushs séparés par de courtes périodes de repos. Sous la même intensité lumineuse, mais à $18^{\circ} \mathrm{C}$ et à $12^{\circ} \mathrm{C}$, le rythme se maintient. Seules les -durées des périodes de croissance et d'arrêt varient. Les caractéristiques morphologiques des premières étapes du développement ont été analysées avec précision, ce qui rend possible la recherche des mécanismes de la rythmicité (Barnola et al, 1986; Champagnat et al, 1986; Alatou et al, 1989; Parmentier et al, 1991).

Obtenir une croissance continue est une stratégie pour comprendre le déterminisme de la croissance rythmique endogène (chêne pédonculé cultivé in vitro: Favre et Juncker, 1989; bruyère cultivée in vitro: Viémont et Beaujard, 1989; feuilles de Guarea guidonia : Miesch, 1990). L'objectif de notre travail se situe dans le cadre de cette stratégie. II faut, avant de le préciser, rappeler que des ob-servations et des études ont été réalisées à ce sujet chez le chêne pédonculé.

Ainsi dans la nature, la croissance continue n'est observable que pour des rejets de souche lors de la première année de leur développement (Lavarenne-Allary, 1965). En conditions expérimentales, à $25^{\circ} \mathrm{C}\left( \pm 1^{\circ} \mathrm{C}\right)$, plusieurs traitements s'opposent à la croissance rythmique :
- l'alternance lumière/obscurité (Lavarenne, 1969);

- les ablations de feuilles très jeunes ayant moins de $10 \mathrm{~mm}$ de longueur (Champagnat et al, 1986; Barnola et al, 1990);

- l'apport d'une cytokinine exogène, la 6benzylaminopurine (Champagnat et al, 1986; Parmentier et al, 1991).

Notre étude reprend et complète les premiers travaux mettant en jeu l'alternance lumière/obscurité (Lavarenne, 1969). Plus précisément, le problème est de savoir comment la modulation de l'intensité lumineuse agit sur la croissance rythmique du chêne pédonculé. Nos installations ne nous permettant pas d'analyser l'influence qualitative de la lumière ni d'étudier réellement l'effet du seul jour court, nous n'avons abordé qu'un aspect restreint mais essentiel et maîtrisé de l'action de la lumière. L'étude apporte de nouvelles précisions sur les expressions du rythme de croissance. Elle renforce l'hypothèse que le déterminisme du rythme est le résultat de corrélations à longue et à courte distances, provenant du fonctionnement de différents organes ou territoires cellulaires plus ou moins éloignés les uns des autres.

\section{MATÉRIEL ET MÉTHODES}

\section{Matériel végétal}

Les plantes sont cultivèes à partir de semences de chêne pédonculé (Quercus robur $L$ ) provenant d'un même semencier.

\section{Méthodes}

\section{Cultures}

Les lots de semences débarrassées de leur péricarpe sont mis à germer. Une sélection est ensuite opérée de façon à avoir des plants homo- 
gènes. Sont repiquées et mises en expérience toutes les germinations dont la radicule a la même longueur (7 à $8 \mathrm{~cm}$ ).

Les plantes sont cultivées sur un substrat de sable grossier à base de quartz et de tourbe brune neutre, mélangés en proportions égales. Elles reçoivent régulièrement, 2 fois par semaine, de la solution nutritive (solution INRAMorizet, tableau I).

L'humidité relative des chambres est maintenue à $80 \%$ et la température à $25^{\circ} \mathrm{C}\left( \pm 1^{\circ} \mathrm{C}\right)$.

Les réserves cotylédonnaires des plants sont épuisées au début du développement de la seconde vague de croissance.

\section{Protocoles expérimentaux}

\section{Culture à l'obscurité}

En l'absence de toute photosynthèse, il est possible de maintenir en croissance pendant 3 semaines les plantes qui se développent grâce aux réserves cotylédonnaires.

Les mesures sont réalisées en lumière verte non active photosynthétiquement et n'agissant pas sur le phytochrome.

\section{Transferts à l'obscurité de plantes d'abord cultivées à la lumière en jour long de $16 \mathrm{~h}$ \\ et retour dans les conditions initiales}

L'intensité lumineuse est de $81 \mu \mathrm{E} \cdot \mathrm{m}^{-2} \cdot \mathrm{s}^{-1}$ à la base des plantes. Elle émane de 29 tubes lumière du jour TF65L et de 12 lampes à incandescence $(16 \mathrm{~W})$ répartis sur une surface de $6 \mathrm{~m}^{2}$.
Les transferts à l'obscurité ont lieu lorsque les plantes abordent la deuxième vague de croissance à des stades de développement bien précis :

- lorsque les feuilles à limbe assimilateur sont épinastiques et anthocyanées, au $7^{e}-8^{e}$ jour de la vague de croissance. La pousse mesure 30 à $40 \mathrm{~mm}$ (stade 1);

- pendant la phase de repos correspondant à un arrêt d'élongation de la tige, au $16^{\mathrm{e}}$ jour de la vague de croissance. Les feuilles à limbe avorté desséché forment les premières écailles d'un bourgeon de $1 \mathrm{~mm}$ dans lequel l'organogenèse se poursuit (stade 2).

\section{Transferts de plantes initialement cultivées à l'obscurité sous des intensités lumineuses de moyenne et faible intensités}

Des intensités lumineuses faibles de 5 et 1,8 $\mu \mathrm{E} \cdot \mathrm{m}^{-2} \cdot \mathrm{s}^{-1}$ sont obtenues en interposant entre la source lumineuse et les plantes un tissu translucide. Le tissu n'affecte pas les qualités spectrales de la lumière blanche initiale, d'intensité moyenne de $81 \mu \mathrm{E} \cdot \mathrm{m}^{-2} \cdot \mathrm{s}^{-1}$.

Les cultures sont réalisées d'abord à l'obscurité jusqu'à la fin de la première vague de croissance et transférées dès le début de la deuxième vague dans les conditions indiquées précédemment.

\section{Culture sous forte intensité lumineuse}

Une intensité de $420 \mu \mathrm{E} \cdot \mathrm{m}^{-2} \cdot \mathrm{s}^{-1}$ est procurée par un éclairement blanc obtenu à partir de 6 lampes à iodure métallique. La durée d'éclairement est de $16 \mathrm{~h}$.

Tableau I. Composition de la solution nutritive INRA-Morizet.

\begin{tabular}{|c|c|c|c|c|c|c|c|}
\hline \multirow{2}{*}{$\begin{array}{l}\text { meq/l } \\
\mathrm{K}^{+}\end{array}$} & \multirow{2}{*}{$\frac{\mathrm{NO}_{3}^{-}}{1,0}$} & \multicolumn{2}{|c|}{ DIH.P ${ }^{P O_{4}^{3-}}$ HYD.P } & \multirow{2}{*}{$\begin{array}{c}\mathrm{SO}_{4}^{2-} \\
-\end{array}$} & \multirow{2}{*}{$\begin{array}{c}C+ \\
-\end{array}$} & \multicolumn{2}{|c|}{ Total cations } \\
\hline & & 4,85 & 0,51 & & & & 6,35 \\
\hline $\mathrm{Na}^{+}$ & - & - & - & - & 0,09 & & 0,09 \\
\hline $\mathrm{Ca}^{2+}$ & 1,46 & - & - & - & - & & 1,46 \\
\hline $\mathrm{Mg}^{2+}$ & - & - & - & 1,10 & - & & 1,10 \\
\hline $\mathrm{NH}_{4}^{+}$ & - & - & - & 0,55 & - & & 0,55 \\
\hline $\mathrm{H}^{+}$ & - & 9,7 & 0,25 & - & - & & 9,95 \\
\hline Total anions & 2,46 & 15,31 & 1,65 & 0,09 & & 19,5 & 19,5 \\
\hline
\end{tabular}




\section{Mesures de croissance}

Les paramètres pris en compte permettent d'apprécier :

- la croissance en longueur de l'axe et des entre-nœuds;

- le nombre d'ensembles foliaires dégagés par unité de temps (plastochrone apparent);

- les durées des phases de repos et de croissance.

\section{Étude biochimique : utilisation de la DMO}

La technique consiste à évaluer par la mesure de la radioactivité la pénétration dans les cellules d'un acide faible lipophile, la 5-5' diméthyloxazolidine-2,4-dione $\left[2^{-14} \mathrm{C}\right]$ ou $\mathrm{DMO}$, de pKa 6,3. La sonde traverse les membranes uniquement sous sa forme non dissociée. Elle se dissocie à l'intérieur et à l'extérieur de la cellule selon le $\mathrm{pH}$ de chaque compartiment jusqu'à l'obtention d'un équilibre de diffusion. La technique fut mise au point sur des cultures de cellules d'érable isolées (Kurkdjian et Guern, 1978) et adaptée aux parenchymes de topinambour (Gendraud et Lafleuriel, 1983), de crosne du Japon (Auriac, 1987) et finalement aux tissus des végétaux ligneux (chêne pédonculé : Barnola et al, 1986; Alatou et al, 1989; Châtaigner: Pezet-Si-Mohamed, 1987).

L'intérêt de la technique est de caractériser les potentialités de croissance des territoires cellulaires. L'accumulation de la DMO dans un territoire cellulaire plus que dans un autre reflète des capacités plus importantes à mobiliser des nutriments (Pezet-Si-Mohamed, 1987).

L'étude biochimique est réalisée au cours de la première vague de croissance sur des plants cultivés à l'obscurité. Les stades examinés correspondent à des prélèvements qui ont lieu tous les 6 jours. Les territoires étudiés sont:

- le bourgeon apical débarrassé de ses écailles;

- un morceau d'axe sous-jacent au bourgeon apical. Les tissus sont essentiellement constitués de parenchymes médullaire et cortical;

- des fragments de feuilles prélevées au niveau moyen de l'étage.

Le rapport de la concentration intracellulaire (Ci) à la concentration extracellulaire (Ce) en $\left[{ }^{14} \mathrm{C}\right]$-DMO est déterminé pour chaque échan- tillon. Les dosages sont effectués pour chaque stade sur 16 plantes.

\section{Expression des résultats}

Les cinétiques de croissance des parties aériennes d'un individu représentatif de l'échantillon sont figurées par des courbes d'évolution de la longueur totale de l'axe principal au cours du temps.

L'organisation spatiale des parties aériennes, pour un individu représentatif de l'échantillon, est représentée sous forme de diagrammes mettant en regard les ensembles foliaires constitutifs des vagues de croissance et la longueur des entre-nœuds qui leur sont associés.

L'individu représentatif sélectionné est celui dont les caractéristiques de croissance sont les plus proches des caractéristiques moyennes de l'échantillon.

Les résultats concernant la composition des vagues et les périodes d'allongement et de repos sont traités par des moyennes associées à un intervalle de confiance au seuil de sécurité de $95 \%$ calculés par la méthode statistique classique. Le nombre d'individus est précisé pour chaque expérience.

\section{RÉSULTATS}

\section{Culture à l'obscurité}

\section{Étude de la croissance}

Il est possible de maintenir en croissance les germinations pendant environ 3 semaines. Se forment alors le premier étage et le début du second. Le comportement des individus diffère et 2 groupes de plantes sont à distinguer :

- le premier groupe, qui constitue $37 \%$ des glands mis en culture, est caractérisé par une croissance où la limite entre le premier et le second étage est seulement marquée par un entre-nœeud court et une feuille plus réduite que les autres, mais entière (fig 1a). La période de repos normalement 

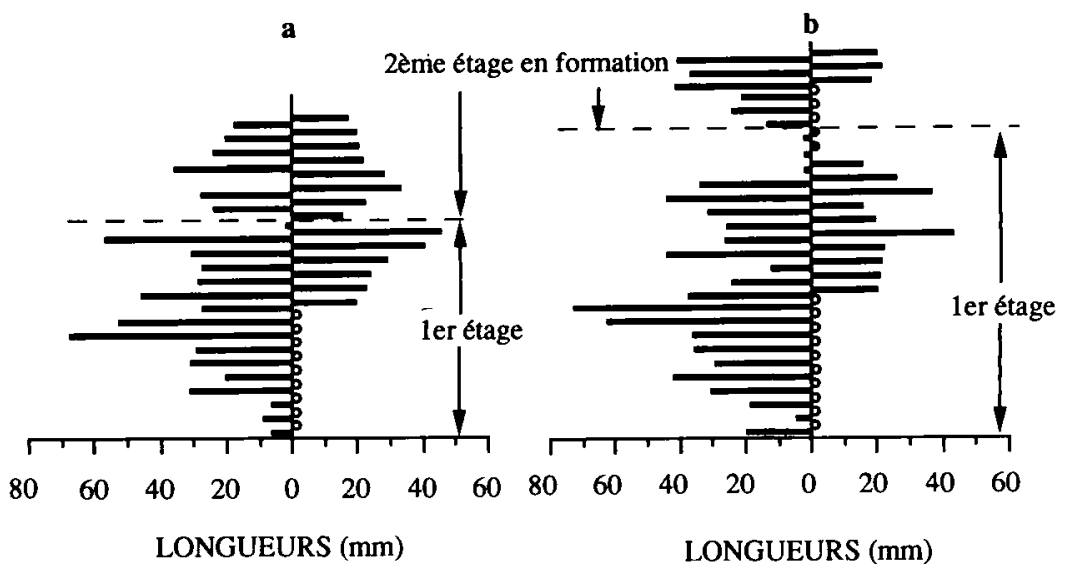

Entre-noeuds Feuilles

Entre-noeuds

Feuilles
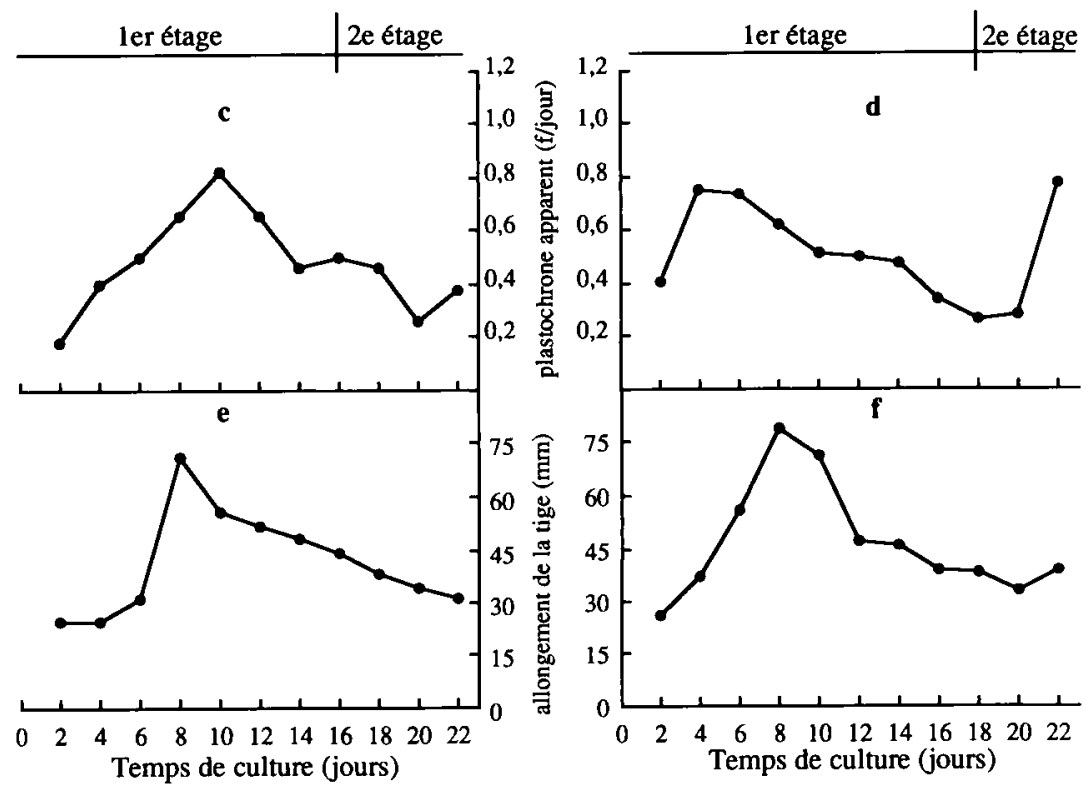

Fig 1. a et b. Représentation schématique de deux plants de chêne pédonculé cultivés à l'obscurité à $25^{\circ} \mathrm{C} \pm 1^{\circ} \mathrm{C}$ ). : feuilles à limbe avorté; $\mathrm{O}$ : stipules écailleuses. $\mathrm{c}$ et $\mathrm{d}$. Évolution des plastochrones apparents en feuilles par jour chez le chêne pédonculé cultivé à l'obscurité à $25^{\circ} \mathrm{C}\left( \pm 1^{\circ} \mathrm{C}\right)$. e et $\mathrm{f}$. Évolution de l'allongement caulinaire en $\mathrm{mm}$ chez le chêne pédonculé cultivé à l'obscurité à $25^{\circ} \mathrm{C}$ $\left( \pm 1^{\circ} \mathrm{C}\right)$. a, $c$ et $\mathrm{e}$ : plant où le passage entre la première et la deuxième vague de croissance se fait par des limbes entiers. $b$, $d$ et $f$ : plant où le passage entre la première et la deuxième vague de croissance se fait par des écailles. 
observée entre les 2 premières vagues de croissance chez les plants cultivés à la lumière est abolie. La vitesse d'allongement n'est jamais nulle pendant tout le temps de la culture. II n'y a pas de formation d'écailles caractérisant l'hétéroblastie, comme cela est le cas à la lumière. Le plastochrone apparent suit une courbe ascendante du $2^{\mathrm{e}}$ au $10^{\mathrm{e}}$ jour de la culture où il atteint 0,8 feuille par jour (fig 1c). II s'abaisse ensuite pour atteindre 0,4 feuille par jour aux $14^{\mathrm{e}}$ et $18^{\mathrm{e}}$ jours. L'allongement est maximal au $8^{e}$ jour, il diminue rapidement entre le $8^{e}$ et le $12^{e}$ jour, plus faiblement ensuite (fig 1e);

- le second groupe se distingue par davantage d'entre-ncuds et par la présence de feuilles réduites à des écailles (fig 1b). En fait, il correspond à des plantes où la première vague de croissance possède 4 ou 5 entre-nœuds en plus, autrement dit dont le développement est prolongé. II n'y a pas d'arrêt de croissance en longueur des plantes comme pour le groupe précédent. La courbe d'évolution des plastochrones apparents est différente de celle du premier groupe. En particulier le maximum, 0,8 feuille par jour, est atteint dès le $4^{\mathrm{e}}$ jour. La décroissance est ensuite beaucoup plus lente que pour le groupe précédent : respectivement 0,5 et 0,3 feuille par jour aux $14^{\mathrm{e}}$ et $18^{\mathrm{e}}$ jours de la culture (fig 1d). L'allongement des entre-nœuds est dans l'ensemble plus important (fig 1f).

\section{Pénétration de la DMO}

Le bourgeon terminal est le territoire qui présente la capacité de rétention intracellulaire en $\left[{ }^{14} \mathrm{C}\right]-\mathrm{DMO}$ la plus élevée. Le rapport de la concentration intracellulaire à la concentration extracellulaire $\left(\mathrm{C}_{i} / \mathrm{C}_{e}\right)$ se maintient en moyenne à la même valeur pendant presque tout le temps de la culture jusqu'au $18^{\mathrm{e}}$ jour (fig 2). Les tissus de l'axe situés sous le bourgeon terminal ne

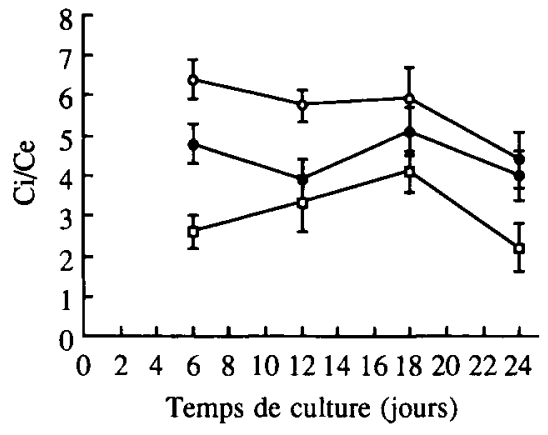

Fig 2. Évolution du rapport de la concentration intracellulaire à la concentration extracellulaire en DMO $\left(C_{i} / C_{e}\right)$ chez le Chêne pédonculé cultivé à l'obscurité à $25^{\circ} \mathrm{C}\left( \pm 1^{\circ} \mathrm{C}\right)$. $\mathrm{O}$ : bourgeon apical; $\bullet$ : axe sous-jacent au bourgeon; [] : limbe entier. Chaque valeur représente la moyenne de 16 mesures \pm erreur standard.

parviennent jamais à égaler ou à dépasser les valeurs $C_{i} / C_{e}$ en $D M O$ relevées pour le bourgeon terminal, comme cela se produit à la lumière (Alatou et al, 1989). L'écart ne se comble qu'à la fin de la culture, lorsque les différences morphologiques ont été mises en place. Les feuilles sont les ensembles qui retiennent le moins la DMO. Leur capacité intracellulaire de rétention de la DMO augmente néanmoins régulièrement du $6^{\mathrm{e}}$ au $18^{\mathrm{e}}$ jour de la culture, ce qui correspond à la durée de leur phase d'expansion. Elle s'abaisse dans la dernière période de la culture quand leur croissance a cessé.

Ainsi, l'obscurité favorise la mise en place d'une croissance continue mais, alors que 2 groupes de plantes sont observés en prenant en compte différents paramètres de la croissance, avec la technique biochimique utilisée, cette différence n'apparaît pas. 


\section{Transferts à l'obscurité \\ de plantes cultivées en jour long et retour dans les conditions initiales}

Ils concernent des plantes ayant achevé leur première vague de croissance à la lumière. Aux 2 stades, stade 1 et stade 2, du développement de la pousse, les comportements observés sont opposés (tableau II).

\section{Plantes transférées à l'obscurité au stade feuilles épinastiques, au $8^{e}$ jour de la vague de croissance : stade 1}

Elles achèvent sans changement apparent leur deuxième vague de croissance débutée à la lumière. Les entre-nœuds sont un peu plus longs à la suite de la croissance à l'obscurité. Le changement notable concerne la phase de repos marquant la fin de cette deuxième vague de croissance: elle est de 4 jours en moyenne. Après retour à la lumière où s'effectue la troisième vague de croissance, le repos entre la troisième et la quatrième vague est aussi moins long, 6 jours au lieu de 8 chez les témoins. L'organogenèse n'est pas affectée par ce second transfert. II n'y a aucune modification de la composition foliaire.

\section{Plantes transférées à l'obscurité pendant la phase de repos séparant la première de la deuxième vague de croissance, au $18^{e}$ jour de la vague de croissance : stade 2}

La phase de repos est considérablement prolongée : de 8 jours chez les plantes restées à la lumière, elle passe à 29 jours. Après retour à la lumière, à la fin de ce repos, la troisième vague de croissance n'est en rien modifiée par rapport à celle des témoins. Croissance en longueur et différenciation foliaire sont comparables. En revanche, la période de repos qui la suit est augmentée de 13 jours. Comme dans le cas précédent, une modification concernant la durée du repos est consécutive au traitement.

Table II. Durées des phases d'allongement et de repos des deuxième et troisième vagues de croissance de plants de chêne transférés à l'obscurité puis remis dans les conditions témoins $\left(25^{\circ} \mathrm{C}\right.$ $\left( \pm 1^{\circ} \mathrm{C}\right.$ ), jour long) lorsqu'ils sont au stade feuilles épinastiques (stade $1: 8^{\mathrm{e}}$ jour de croissance de la deuxième vague $=8^{\mathrm{ej}} 2^{\mathrm{e}} \mathrm{v}$ ) et au stade mi-repos apparent (stade $2: 18^{\mathrm{e}}$ jour de croissance de la deuxième vague $=18 \mathrm{e} 2^{2} \mathrm{v}$ ). $\mathrm{n}$ représente le nombre d'individus observés pour chaque traitement.

$\frac{\begin{array}{c}2 \text { vague de croissance } \\ \text { Durées en jours }\end{array}}{\text { Allgt Repos }} \frac{\begin{array}{c}3^{e} \text { vague de croissance } \\ \text { Durées en jours }\end{array}}{\text { Allgt Repos }}$

Témoin cultivé à $25^{\circ} \mathrm{C}$, jour long $\quad 10,3 \pm 0,5 \quad 8,6 \pm 0,5 \quad 12,5 \pm 0,6 \quad 8,8 \pm 0,7$

Stades morphologiques de transfert à l'obscurité
Stade $1: 8^{e_{j}} 2^{e} v$ (feuilles épinastiques)
$12,1 \pm 1,4 \quad 4,2 \pm 1,6$
$12,8 \pm 2,2$
$6,0 \pm 0,9$
$n=11$
$9,7 \pm 0,5 \quad 29,3 \pm 3,4 \quad 7,4 \pm 1,6 \quad 20,7 \pm 2,5$
Stade $2: 18^{e} \mathrm{j} 2^{e} \vee($ mi-repos apparent)
$n=14$ 


\section{Transferts à la lumière de chênes cultivés initialement à l'obscurité}

Le passage de plantes cultivées d'abord à l'obscurité à des intensités lumineuses faibles (5 et $1,8 \mu \mathrm{E} \cdot \mathrm{m}^{-2} \cdot \mathrm{s}^{-1}$ ) ou comparables aux témoins $\left(81 \mu \mathrm{E} \cdot \mathrm{m}^{-2} \cdot \mathrm{s}^{-1}\right)$ conduit dans tous les cas au rétablissement de l'arrêt de croissance bien distinct entre 2 vagues.

Les transferts ainsi réalisés entraînent une modification des temps d'allongement, des temps de repos et beaucoup moins de la différenciation foliaire (tableaux III et IV).

\section{Transfert à $81 \mu \mathrm{E} \cdot \mathrm{m}^{-2} \cdot \mathrm{s}^{-1}$}

En 3-4 jours, les plantes terminent leur allongement, ce qui conduit à la formation d'un second étage réduit en longueur, mais où les limbes assimilateurs qui lui sont associés grandissent normalement.

La durée de l'allongement et les caractéristiques foliaires de la troisième vague

Tableau III. Expressions temporelles des deuxième et troisième vagues de croissance de jeunes plants de chêne pédonculé cultivés initialement à l'obscurité à $25^{\circ} \mathrm{C}\left( \pm 1^{\circ} \mathrm{C}\right)$, et transférés à la lumière sous différentes intensités lumineuses $: i_{1}=81 \mu \mathrm{E} \cdot \mathrm{m}^{-2} \cdot \mathrm{s}^{-1} ; \mathrm{i}_{2}=5 \mu \mathrm{E} \cdot \mathrm{m}^{-2} \cdot \mathrm{s}^{-1} ; i_{3}=1,8 \mu \mathrm{E} \cdot \mathrm{m}^{-2} \cdot \mathrm{s}^{-1}$. Les transferts ont lieu lorsque les témoins cultivés à $25^{\circ} \mathrm{C}\left( \pm 1^{\circ} \mathrm{C}\right)$, sous une intensité lumineuse de 81 $\mu \mathrm{E} \cdot \mathrm{m}^{-2} \cdot \mathrm{s}^{-1}$, démarrent leur deuxième vague de croissance. $n$ représente pour chaque traitement le nombre d'individus observés.

\section{$2^{e}$ vague de croissance Durée en jour de la phase de repos}

$3^{e}$ vague de croissance

Durée en jours de la phase d'allongement de la phase de repos

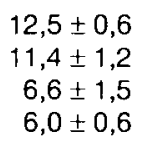

$8,8 \pm 0,7$

$18,0 \pm 3,2$

$36,5 \pm 5,5$

$17,5 \pm 1,2$

Tableau IV. Expressions spatiales des deuxième et troisième vagues de croissance de jeunes plants de chêne pédonculé cultivés initialement à l'obscurité à $25^{\circ} \mathrm{C}\left( \pm 1^{\circ} \mathrm{C}\right)$, et transférés à la lumière sous différentes intensités lumineuses : $i_{1}=8 \mu \mathrm{E} \cdot \mathrm{m}^{-2} \cdot \mathrm{s}^{-1} ; i_{2}=5 \mu \mathrm{E} \cdot \mathrm{m}^{-2} \cdot \mathrm{s}^{-1} ; i_{3}=1,8 \mu \mathrm{E} \cdot \mathrm{m}^{-2} \cdot \mathrm{s}^{-1}$. Les transferts ont lieu lorsque les témoins cultivés à $25^{\circ} \mathrm{C}\left( \pm 1^{\circ} \mathrm{C}\right)$, sous une intensité lumineuse de 81 $\mu \mathrm{E} \cdot \mathrm{m}^{-2} \cdot \mathrm{s}^{-1}$, démarrent leur deuxième vague de croissance. $\mathrm{n}$ représente pour chaque traitement le nombre d'individus observés.

\section{2 vague de croissance Nombre de Nombre de $3^{e}$ vague de croissance paires d'écailles feuilles assimilatrices paires d'écailles feuilles assimilatrices}

$\begin{array}{lllll}\text { Témoin }(n=20) & 3,1 \pm 0,5 & 6,6 \pm 1,0 & 3,3 \pm 0,8 & 6,5 \pm 1,9 \\ i_{1}=81(n=12) & 2,0 \pm 0,4 & 6,7 \pm 1,3 & 3,3 \pm 0,4 & 6,2 \pm 1,0 \\ i_{2}=5(n=11) & - & - & 8,0 \pm 1,2 & 4,0 \pm 1,2 \\ i_{3}=1,8(n=14) & - & - & 4,7 \pm 0,4 & 3,6 \pm 0,4\end{array}$


sont proches de celles des témoins cultivés sous la même intensité. En revanche, les périodes de repos de la deuxième et de la troisième vagues sont allongées, respectivement de 3 et de 9 jours.

\section{Transfert à $5 \mu \mathrm{E} \cdot \mathrm{m}^{-2} \cdot \mathrm{s}^{-1}$}

Les plantes arrêtent leur allongement au bout de 5 jours. La période d'allongement de la troisième vague est raccourcie, elle est de $6,6 \pm 1,5$ jours et les temps de repos de la deuxième et de la troisième vague sont considérablement allongés : $46,4 \pm 12,3$ jours et $36,5 \pm 5,5$ jours. Du point de vue différenciation foliaire, nous notons une augmentation du nombre d'écailles et une réduction du nombre de limbes assimilateurs pour la troisième vague de croissance.

\section{Transfert à 1,8 $\mu \mathrm{E} \cdot \mathrm{m}^{-2} \cdot \mathrm{s}^{-1}$}

L'allongement est suspendu au bout de 6 jours. Les périodes de repos de la deuxième et de la troisième vagues de croissance sont prolongées de 13-14 jours et de 9 jours. Le temps d'allongement de la troisième vague est, comme à $5 \mu \mathrm{E} \cdot \mathrm{m}^{-2} \cdot \mathrm{s}^{-1}$, de $6 \pm 0,6$ jours, ce qui conduit aussi à un étage de longueur réduite. Pour cette dernière vague, le nombre d'écailles est augmenté. Cette situation va de pair avec une diminution du nombre de limbes assimilateurs.

En résumé, il faut retenir, en premier lieu, que les transferts à la lumière permettent dans tous les cas, pour tous les plants, le rétablissement de la croissance rythmique. Elle se manifeste même sous une intensité lumineuse très faible. En second lieu, il faut retenir que l'expression temporelle du rythme est plus affectée par ces traitements que l'expression spatiale.

\section{Culture sous forte intensité lumineuse $\left(420 \mu E \cdot m^{-2} \cdot \mathrm{s}^{-1}\right)$}

La croissance rythmique endogène n'est pas modifiée par rapport à celle obtenue sous une plus faible intensité lumineuse à $81 \mu \mathrm{E} \cdot \mathrm{m}^{-2} \cdot \mathrm{s}^{-1}$. Le changement notable touche la morphogenèse du plant car il se manifeste une atténuation de la dominance apicale. En effet, pour $70 \%$ des plants, le développement du bourgeon apical s'accompagne de celui des bourgeons axillaires les plus proches de lui. Si la croissance des ramifications est freinée par le développement de l'axe principal, elle reste rythmique. Une première vague de croissance est synchrone de celle de l'axe principal, la suivante est plus tardive du fait de l'allongement du temps de repos (fig 3). Les ramifications ont un développement limité par rapport à celui de la pousse principale et l'acrotonie, bien qu'amoindrie, est conservée.

Au niveau des ramifications, le nombre de feuilles à limbe assimilateur et la longueur de la tige sont significativement plus faibles que ceux de la tige principale.

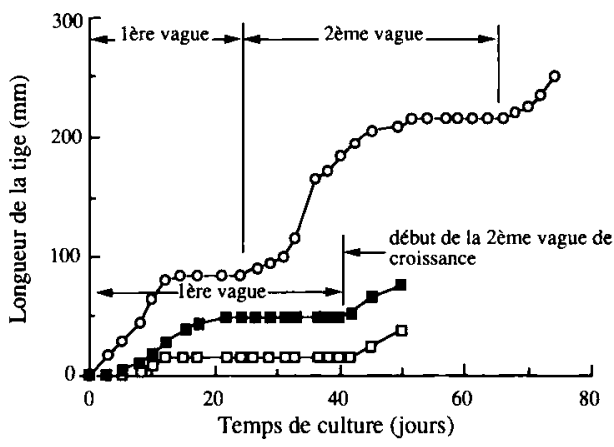

Fig 3. Évolution de la longueur de la tige $(\mathrm{O})$ et des longueurs de 2 axillaires sous-jacents au bourgeon terminal ( $[\square$ et $\mathbf{C}$ ) d'un plant de chêne pédonculé .cultivé à $25^{\circ} \mathrm{C}\left( \pm 1^{\circ} \mathrm{C}\right)$, en jour long de $16 \mathrm{~h}$, sous une intensité lumineuse de $420 \mu \mathrm{E} \cdot \mathrm{m}^{-2} \cdot \mathrm{s}^{-1}$. 
Enfin, au niveau de l'axe principal, le nombre de feuilles assimilatrices et leur taille augmentent dès la deuxième vague de croissance. De même, la durée de la phase d'allongement est augmentée, ce qui contribue à une élévation de la durée totale de la vague de croissance.

\section{DISCUSSION}

À notre connaissance, seuls les travaux de Lavarenne (1969) ont posé le problème des rapports entre la croissance rythmique endogène et l'action de la lumière. Depuis, aucune publication n'est parue sur le sujet bien que des situations d'entrée en dormance soient décrites avec des espèces ayant une croissance rythmique aussi typique que celle du chêne lorsque le jour diminue en longueur et en intensité (Beaujard et al, 1991).

La condition de culture qui permet une croissance continue est l'obscurité, ce qui confirme les observations de LavarenneAllary (1969). Il est alors nécessaire de préciser quelles sont les caractéristiques physiologiques qui la rendent possible et qui n'ont, jusqu'à présent, jamais été analysées. Depuis longtemps, on sait que c'est à la lumière que se manifeste pleinement la croissance rythmique endogène (Klebs, 1917; Lavarenne-Allary, 1965). II apparaît donc que la modulation de son intensité puisse renseigner sur ces mécanismes. Aussi nous faut-il, au cours de cette étude, répondre aux questions suivantes :

- lorsqu'un passage à l'obscurité a lieu, peut-il favoriser une croissance continue et quelle expression de la croissance rythmique est alors modifiée?

- est-ce que la croissance rythmique se réalise quelle que soit l'intensité lumineuse choisie? Quels sont donc les comporte- ments des plantes à faibles et à fortes intensités lumineuses ?

- en quoi la modulation de l'intensité lumineuse permettrait-elle de mieux connaître les mécanismes de la croissance rythmique?

\section{À l'obscurité, le bourgeon terminal est le territoire dominant, contrairement à ce qui se passe à la lumière. La croissance rythmique est déstabilisée, elle devient continue}

Deux groupes de plantes sont observés, qui ont en commun la suppression de la période de repos séparant la première vague de la deuxième. Ces repos correspondent, en fait, à la lumière, à une suspension temporaire des capacités à s'allonger des entre-nœuds préformés dans le bourgeon terminal. Autrement dit, à l'obscurité l'allongement est ininterrom$\mathrm{pu}$, seule varie sa vitesse. Dans le premier groupe, la limite entre le premier et le deuxième flush est marquée seulement par une diminution de longueur de la feuille dont l'entre-nœud sous-jacent ne s'allonge pas. Dans l'autre groupe, la vague initiale a une croissance prolongée et se termine par la mise en place d'ensembles écailleux associés à des entrenœuds très courts.

Bien que les 2 expressions du rythme soient modifiées, la plus touchée est l'expression temporelle. L'expression spatiale peut encore se manifester, comme dans le second groupe. Elle résulterait de corrélations intra-apicales selon un schéma développé par Fulford (1965), Neville (1968), Champagnat et al (1986). Ainsi, la transformation d'une feuille à limbe entier en une feuille écailleuse serait la conséquence de l'accumulation des ébauches foliaires dans un bourgeon, concomitante d'une impossibilité d'allonge- 
ment des entre-nœuds. À l'obscurité, cette situation serait modifiée, l'allongement est privilégié et l'inhibition de la différenciation en feuilles à limbes entiers conduisant à des ensembles écailleux est partiellement levée.

L'interprétation des faits observés pourrait être envisagée, mais les expériences restent à faire, en terme d'allocation en glucides solubles provenant des réserves cotylédonnaires. Cette allocation est à la base des disponibilités énergétiques au niveau des ensembles en croissance et en différenciation constitutifs de l'axe aérien. À l'obscurité, la prédominance du bourgeon terminal est mise en évidence par la $\left[{ }^{14} \mathrm{C}\right]$-DMO. L'intérêt de la DMO en tant que marqueur de territoires cellulaires aptes à la croissance, voire de puits, a été souligné à plusieurs reprises dans des situations de dormance (Gendraud et Lafleuriel, 1983; Auriac, 1987; PezetSi-Mohamed, 1987; Candelier, 1989) et lors de l'étude de la croissance rythmique endogène (Alatou et al, 1989; Barnola et al, 1990; Parmentier et al, 1991). À l'obscurité, la seule source de nutriments organiques est constituée par les réserves cotylédonnaires. Les résultats obtenus avec la DMO montrent qu'ils devraient être utilisés au niveau du bourgeon terminal, plus qu'au niveau des autres ensembles examinés, feuilles et portion d'axe sousjacente au bourgeon terminal. À la lumière, nous avions montré (Alatou et al, 1989) que la situation est tout autre: les feuilles et les tissus de l'axe dépassent le bourgeon terminal en possibilités de rétention intracellulaire de la DMO, ils le concurrenceraient donc au plan trophique. Au contraire, à l'obscurité la prédominance du fonctionnement gemmaire étant privilégiée, l'extension des entrenœuds initiés serait toujours possible et serait à la base de la croissance continue observée.

\section{Les expériences de transfert à l'obscurité à $25^{\circ} \mathrm{C}\left( \pm 1^{\circ} \mathrm{C}\right)$ de plantes cultivées initialement en jour long, à la même température, avec retour dans les conditions initiales, montrent que seule l'expression temporelle du rythme est profondément affectée}

Le résultat montre la stabilité de l'expression spatiale et les possibilités de modulation de l'expression temporelle. Lorsque les plantes entrent en repos, le passage à l'obscurité provoque un allongement important de ce repos. En revanche, le même traitement effectué quand les plantes entament une nouvelle vague de croissance raccourcit le temps de repos. La prolongation est beaucoup plus importante que le raccourcissement obtenu. Le temps de repos par rapport à des témoins toujours cultivés à la lumière est, dans un cas, multiplié par un facteur 3 à 4 alors qu'il est divisé par un facteur 2 dans l'autre cas. L'effet est d'autant plus remarquable que la phase de repos de la vague suivante, qui intervient entièrement à la lumière, est modifiée dans le même sens. Les 2 stades auxquels sont effectués les traitements ne sont séparés que par 6 jours, ce qui prouve que l'état physiologique des plantes évolue très rapidement au cours de la vague de croissance. La tendance à une croissance continue par diminution du temps d'arrêt de l'élongation a lieu lorsque la plante aborde une nouvelle phase de croissance, au moment où l'accessibilité des nutriments au bourgeon est la plus forte (Barnola et al, 1986).

De plus, les 2 stades où les transferts ont lieu, au plan de l'énergétique cellulaire, ont des caractéristiques opposées (Barnola et al, 1986; Alatou et al, 1989). En effet, au début du repos, le bourgeon terminal présente une difficulté à synthétiser des composés adényliques et non adényli- 
ques. À l'inverse, les capacités pour cette synthèse sont fortes lors de la reprise de croissance de la vague. II est vraisemblable que l'énergétique cellulaire soit en rapport avec les variations de l'allocation des glucides. II est donc logique de penser à un rapport de la croissance rythmique avec les glucides. L'hypothèse avait été évoquée par Champagnat et al (1968) et le développement de nouvelles techniques biochimiques d'étude permettent actuellement de mieux la préciser.

\section{Les transferts à la lumière, au début du développement de la deuxième vague de croissance, de chênes pédonculés cultivés à l'obscurité, montrent que de très faibles intensités lumineuses restaurent la croissance rythmique}

Le transfert à la lumière, sous des intensités de 5 et $1,8 \mu \mathrm{E} \cdot \mathrm{m}^{-2} \cdot \mathrm{s}^{-1}$ entraîne une augmentation du temps de repos, la réduction en longueur du troisième étage, un accroissement du nombre d'écailles et la diminution du nombre de limbes assimilateurs de ce troisième étage. Lorsque le retour à la lumière a lieu dans les conditions de culture des témoins, sous une intensité de $81 \mu \mathrm{E} \cdot \mathrm{m}^{-2} \cdot \mathrm{s}^{-1}$, aucune des modifications précédentes n'est notée. La croissance est similaire à celle des témoins, excepté un raccourcissement en longueur de l'étage obtenu, sans modification de la succession entre-nœuds longs/entrenœuds courts. Les relations à l'allocation glucidique et à l'énergétique cellulaire sont à l'étude.

Le fait que la croissance rythmique du chêne se manifeste sous un éclairement de très faible intensité serait à approfondir, en liaison avec la qualité de la lumière et notamment par rapport aux radiations rouge clair et rouge sombre, comme cela a été réalisé dans l'étude de la croissance rythmique d'autres espèces ligneuses (Terminalia superba : Maillard et al, 1987; Ginkgo biloba: Flesch et Jacques, 1991).

Au total, la lumière, non seulement en intensité mais aussi en durée et vraisemblablement en qualité, nous apparaît être un facteur primordial de la croissance du chêne. Cette situation, à notre avis, est en accord avec la manifestation soutenue d'un rythme de croissance endogène qui en fait l'espèce la plus tropicalisée des arbres des zones tempérées (Champagnat et Lamond, 1979). Des travaux anciens ont, en outre, montré que le jour court provoque la suspension de la croissance mais que la reprise du développement intervient dès que des conditions de jour long sont rétablies (Nitsch, 1957). Une remarque est à faire: dans les conditions naturelles, au moment de la période d'entrée en dormance, au niveau du bourgeon terminal du chêne pédonculé, un plus grand nombre d'ensembles foliaires écailleux, jusqu'à 12 en moyenne, est mis en place. II est beaucoup plus élevé que celui observé au niveau de ce même bourgeon entre deux pousses de la Saint-Jean. Cela traduit une inhibition accentuée de la croissance dont les causes sont à rechercher pour une part dans la diminution de l'éclairement. Mais dans les conditions naturelles, il ne faut pas dissocier cet effet d'un autre facteur qui est la diminution des températures. En effet, même lorsque nous avons fortement augmenté le temps de repos à $25^{\circ} \mathrm{C}$, le nombre d'ensembles écailleux ne s'est pas élevé. Par rapport aux conditions naturelles, nous n'avons modulé que l'intensité lumineuse sans jouer sur des variations de températures et notamment sans introduire des températures fraîches ou basses dans le cycle journalier.

Chez des ligneux, des travaux récents montrent l'importance de la longueur de la photopériode dans la modulation de l'expression temporelle du rythme endogène de croissance, que ce soit chez des es- 
pèces où le rythme est très net (bruyère cultivée in vitro : Viémont et Beaujard, 1991; Rhododendron : Beaujard et al, 1991), ou chez des espèces où il est moins évident (Gleditsia : Aillaud et al, 1989). L'approfondissement en cours des mécanismes, par la recherche de marqueurs biochimiques, devrait contribuer à la compréhension de la croissance rythmique endogène et de ses rapports à la dormance (Beaujard et al, 1991).

\section{La culture du chêne pédonculé sous une forte intensité lumineuse n'entraîne pas de modification du rythme endogène de croissance mais provoque une levée partielle de la dominance apicale et la croissance des axillaires proches du bourgeon terminal}

La croissance des axillaires est limitée car la pousse issue du bourgeon terminal reste dominante. La réaction à une forte intensité lumineuse rappelle celle obtenue lorsque les troncs des chênes pédonculés sont, à la suite d'une pratique de sylviculture, brutalement exposés à la pleine lumière (Roussel, 1972). Elle rappelle également la réaction communément observée lorsque des chênes se développent isolément. Nos résultats mettent en évidence l'importance, dans ce processus de levée de la dominance apicale, de la durée et de l'intensité de la lumière. Chez Terminalia superba (Maillard, 1987), des éclairements de forte intensité appliqués sur un temps long donnent des résultats similaires.

En fait, nos cultures reçoivent une solution nutritive assez riche en azote. $\|$ y a très certainement une conjonction entre le niveau nutritionnel et l'éclairement fort qui conduit à la morphogenèse observée. Chez le chêne sessile, des travaux ont en effet montré qu'un apport élevé en azote favorise la mise en place d'une ramification subapicale (Harmer, 1989). L'accent est mis ainsi sur l'importance des régulations trophiques quant à l'expression de la dominance apicale. Les résultats apparaissent proches de ceux analysés classiquement chez les herbacées (Migniac, 1974; Crabbé, 1987). C'est d'ailleurs ce type d'interactions entre facteurs trophiques qui est mis à profit chez des ligneux horticoles pour contrôler leurs formes (rhododendron : Beaujard et al, 1991). L'apparition des ramifications chez un arbre est, selon Edelin (1991), un processus fondamental de son développement. Pour cet auteur, les premières ramifications ne subsistent pas et caractérisent la phase de jeunesse du végétal. Leur conservation serait une étape décisive de l'accomplissement du programme architectural. Selon nous, il n'y a pas de raison de penser que les mécanismes qui conduisent à l'apparition des ramifications ne soient pas les mêmes, qu'elles subsistent ou non, leur pérennité étant un autre problème.

\section{CONCLUSION}

L'étude du rythme endogène de croissance en rapport avec l'influence de la lumière en durée et intensité permet de préciser des caractéristiques physiologiques du rythme, proches de son déterminisme.

Notre approche reste encore partielle puisque d'autres mécanismes explicatifs sont mis en avant avec d'autres végétaux ligneux : gestion de l'eau (hévéa: Hallé et Martin, 1968; cacaoyer : Borchert, 1973; Vogel, 1975), influence de l'azote minéral (bruyère cultivée in vitro : Viémont et Beaujard, 1989; chêne pédonculé cultivé in vitro: Favre et Juncker, 1989), action des régulateurs de croissance (acide abscissique : Mialoundama, 1991; cytokinines : Maillard, 1987), combinaison entre 
des périodes de rythmes endogènes différents pour le système aérien et pour le système racinaire (Millet et al, 1991). II n'est pas de notre propos de discuter dans le cadre de cette publication des différentes hypothèses, mais le rappel que nous en faisons montre finalement que, comme tout processus de développement, la croissance rythmique endogène des végétaux ligneux est un phénomène complexe qui ne pourra être compris que par le développement de voies de recherche diversifiées.

En ce qui nous concerne, les résultats montrent très nettement que les expressions du rythme, temporelle et spatiale, sont influencées par la lumière. Il apparaît finalement que le rythme dépend tout à la fois de corrélations à longue distance intervenant au niveau de l'expression temporelle, et de corrélations à courte distance proches du bourgeon et intraapicales (Champagnat et al, 1986; Barnola et al, 1986, 1990; Alatou et al, 1989). Les corrélations à courte distance extra- et intragemmaires seraient à la base de l'expression spatiale, ce qui pourrait en expliquer la plus forte stabilité observée dans les diverses situations expérimentales testées alors que l'expression temporelle apparaît beaucoup plus sujette à modulation.

Des recherches sont en cours (Parmentier, thèse en cours) pour préciser quel est le rapport entre l'allocation glucidique et les corrélations considérées. En effet, des travaux sur l'alisier (Barnola et Parmentier, 1992) nous font penser qu'elle intervient dans les corrélations à longue distance. L'étude réalisée en faisant varier les intensités lumineuses le suggère également. II serait cependant trop simple de penser qu'une seule catégorie de molécules organiques soit en cause. En réalité, l'essentiel paraît se jouer et cette étude le montre encore, dans l'accessibilité, facilitée ou non, des éléments nécessaires à la croissance au niveau du bourgeon terminal tou- jours apte à les utiliser pour son fonctionnement.

\section{RÉFÉRENCES}

Aillaud G, Al Ibrahem A, Neville P (1989) Influence de la durée de l'exposition en jours courts sur la dormance des bourgeons de Gleditsia triacanthos L. Ann Sci For 46s, 236s-241s

Alatou D, Barnola P, Lavarenne S, Gendraud M (1989) Caractérisation de la croissance rythmique du chêne pédonculé. Plant Physiol Biochem 27(2), 275-280

Auriac MC (1987) Contribution à l'étude de la pénétration des sucres dans les cellules accumulatrices chez le crosne du Japon (Stachys sieboldii Miq). Thèse doctorat université, université de Clermont-Ferrand, $138 \mathrm{p}$

Barnola $P$, Crochet A, Payan E, Gendraud M, Lavarenne S (1986) Modification du métabolisme énergétique et de la perméabilité dans le bourgeon apical et l'axe sous-jacent au cours de l'arrêt de croissance momentané de jeunes plants de chêne. Physiol Vég 24, 307314

Barnola P, Alatou D, Lacointe A, Lavarenne S (1990) Étude biologique de la croissance rythmique du chêne pédonculé (Quercus robur $\mathrm{L}$ ). Effets de l'ablation de feuilles. Ann Sci For 21, 619-631

Barnola P, Durand P, Parmentier C (1993) Recherches préliminaires sur la croissance rythmique endogène et la morphogenèse de l'alisier torminal. Rev For Fr 3, 361-278

Beaujard F, Frustec J, Viémont JD, Denoël C (1991) Développement végétatif chez les rhododendrons ornementaux et contrôle expérimental de la forme. Naturalia monspeliensia hors-série, 397-415

Borchert R (1973) Simulation of rhythmic tree growth under constant conditions. Physiol Plant 29, 173-180

Candelier P (1989) Étude comparée des effets du froid et de l'éthanol sur la dormance de tubercules de topinambour (Helianthus tuberosus L) : évolution de quelques paramètres biochimiques. Thèse université, université de Clermont-Ferrand, $121 \mathrm{p}$ 
Champagnat $P$, Champagnat $M$, Loiseau $M$ (1968) Croissance rythmique du chêne. Film Éditions cinématographiques CNRS

Champagnat $P$, Lamond $L$ (1979) Contribution à l'étude biologique du chêne (Quercus pedunculata Ehrh) et du hêtre (Fagus sylvatica L). Compte rendu de fin d'étude contrat DGRST $n^{\circ}$ 76.7.0543-77.7.1135

Champagnat $P$, Payan $E$, Champagnat $M$, Barnola $P$, Lavarenne S, Bertholon C (1986) La croissance rythmique de jeunes chênes pédonculés cultivés en conditions contrôlées et uniformes. Naturalia monspeliensia horssérie, 303-337

Crabbé J (1987) Aspects particuliers de la morphogenèse caulinaire des végétaux ligneux et introduction à leur étude quantitative. IRSIA, Bruxelles, $116 p$

Edelin C (1991) Nouvelles données sur l'architecture des arbres sympodiaux. Le concept de plan d'organisation. Naturalia monspeliensia hors-série, 127-154

Favre JM, Juncker B (1989) Variations in expression of episodic growth by in vitro cultured shoots of oak (Quercus robur $L$ ). Ann Sci For 46s, 206s-210s

Flesch V, Jacques M (1991) Croissance de jeunes Ginkgo biloba $L$ en conditions contrôlées. Naturalia monspeliensia hors-série, 602-603

Fulford RM (1965) The morphogenesis of apple buds. I. The activity of the apical meristem. Ann Bot 29, 167-180

Gendraud M, Lafleuriel J (1983) Caractéristiques de l'absorption du saccharose et du tétraphénylphosphonium par les parenchymes de tubercules de topinambour (Helianthus tuberosus $\mathrm{L}$ ) dormants et non dormants, cultivés in vitro. Physiol Vég 21, 1125-1133

Hallé F, Martin R (1968) Étude de la croissance rythmique de l'hévéa (Hevea brasiliensis Mull Ay Euphorbiacées crotonoïdés). Adansonia 2-8, 475-503

Harmer R (1989) Some aspects of bud activity and branch formation in young oak. Ann Sci For 46s, 217s-232s

Klebs G (1917) Über das Verhältniss von Wachtum und Ruhe bei den Pflanzen. Biol Zentralb/37, 373-415

Kurkdjian A, Guern J (1978) Intracellular pH in higher cells. Improvement in the use of the 5,5'-dimethyloxazolidine $\left[2-{ }^{14} \mathrm{C}\right]$, 4-dione distribution technics. Plant Sci Lett 11, 337-344

Lavarenne-Allary S (1965) Recherches sur la croissance des bourgeons de chênes et de quelques autres espèces ligneuses. Ann Sci For 22(1), 1-203

Lavarenne S (1969) Déterminisme d'une croissance continue chez le chêne. CR Acad Sci Paris 269, 2099-2102

Maillard P (1987) Étude du développement végétatif du Terminalia superba Englers et Diels en conditions contrôlées : mise en évidence de rythmes de croissance. Thèse, université de Paris-VI, $205 p$

Maillard $P$, Jacques $M$, Miginiac $E$, Jacques $R$ (1987) Croissance de jeunes Terminalia superba en conditions contrôlées. Ann Sci For $44(1), 67-84$

Mialoundama $F$ (1991) Croissance rythmique des systèmes radiculaire et caulinaire chez Gnetum africanum Welch. Naturalia monspeliensia hors-série, 319-325

Miesch R (1990) Morphogenèse de 2 espèces à feuilles composées pennées, Guarea guidonia (L) Sleumer (Méliacée) et Lycopersicum esculentum Miller cv (Solanacée); potentialités exprimées et enseignements phyllogénétiques. Thèse doctorat Sci Nat, université de Strasbourg, $166 \mathrm{p}$

Migniniac E (1974) Quelques aspects morphologiques, physiologiques et biochimiques de la dominance apicale. Physiol Vég 12, 689-720

Millet B, Bonnet B, El-Morsy AW (1991) Le fonctionnement rythmique des végétaux ligneux. Naturalia monspeliensia hors-série, 295-317

Neville P (1968) Morphogenèse chez Gleditsia triacanthos $\mathrm{L}$. I. Mise en évidence expérimentale des corrélations jouant un rôle dans la morphogenèse et la croissance des bourgeons et des tiges. Ann Sci Nat Bot 9, 433510

Nitsch P (1957) Growth responses of woody plants to photoperiodic stimuli. Proc Am Soc Hort Sci 70, 512-525

Parmentier C, Barnola $P$, Maillard P, Lavarenne $S$ (1991) Étude de la croissance rythmique du chêne pédonculé, influence du système racinaire. Naturalia monspeliensia hors-série, 327-343

Pezet-Si-Mohamed Y (1987) Caractérisation des potentialités morphogènes du châtai- 
gnier (Castanea sativa Miller). Distribution et possibilités de translocation des réserves solubles et insolubles associées à des gradients de $\mathrm{pH}$ intracellulaires. Thèse doctorat université, université de Clermont-Ferrand, $62 p+$ fascicule figures

Roussel L (1972) Photologie forestière. Monographies de botanique et de biologie végétale. Masson et Cie, Paris, $132 \mathrm{p}$

Vièmont JD, Beaujard $F$ (1989) Les bruyères in vitro. VIII. Évolution du pH du milieu de cul- ture et morphogenèse de l'Erica $X$ darleyensis (B). Relation entre l'absorption et les phases de la croissance rythmique. Can $J$ Bot 67(1), 161-166

Viémont JD, Beaujard F (1991) Contribution de la culture in vitro d'une bruyère à l'étude de la croissance rythmique. Naturalia monspeliensia hors-série, 672-673

Vogel M (1975) Recherche du déterminisme du rythme de croissance du cacaoyer. Café Cacao Thé 19, 265-290 